\title{
ASO Authors Reflection: Lessons Learned from the COVID-19 Pandemic-Should We Change Surgical Management of Patients with Breast Cancer?
}

\author{
Judy C. Boughey, MD', Toan T. Nguyen, $\mathrm{MD}^{2}$, Jill R. Dietz, $\mathrm{MD}^{3}$, and Lee G. Wilke, $\mathrm{MD}^{4}$ \\ ${ }^{1}$ Department of Surgery, Mayo Clinic, Rochester, MN; ${ }^{2}$ Surgical Oncology, Lakeland Regional Health, Lakeland, FL; \\ ${ }^{3}$ Chief Transformation Officer, Allegheny Health Network Cancer Institute, Pittsburgh, PA; ${ }^{4}$ Department of Surgery, \\ University of Wisconsin School of Medicine and Public Health, Madison, WI
}

\section{PRIOR}

Surgical resection of the primary breast tumor and staging of regional lymph nodes has been the cornerstone in the management of breast cancer for more than 50 years. For the majority of patients, especially those with an estrogen-positive tumor, upfront surgical resection has been the standard approach. Adjuvant systemic and radiation therapy usually followed and were largely determined by surgical procedure, tumor stage, and tumor biology.

\section{CURRENT}

The coronavirus disease 2019 (COVID-19) pandemic resulted in many US health care institutions stopping elective surgery because of an immediate need to conserve resources and to limit patients' exposure to potential COVID-19 risks. This led to the question of whether surgery for breast cancer was truly elective-a question to which most surgeons and patients would answer "no," or, more realistically, "no, not really." However, when faced with a situation that in a highly resourced country we never anticipated, and without any preparation or precedent for it, many surgeons and institutions needed to quickly prioritize and ration surgical care. And the truth is that, for many breast cancer patients [those with estrogen receptor (ER)

(C) Society of Surgical Oncology 2021

First Received: 23 July 2021

Accepted: 30 July 2021

Published Online: 11 August 2021

L. G. Wilke, MD

e-mail: wilke@surgery.wisc.edu positive disease], as opposed to most other solid organ malignancies, providers have the option to bridge these patients safely with neoadjuvant endocrine therapy (NET). As a result, in a time of limited OR access, many patients with ER+ breast cancer were treated with NET. This treatment approach permitted delays in surgical resection until additional personal protective equipment (PPE) and other resources could be obtained and specific COVID-19 hospital protocols implemented. ${ }^{1}$ Genomic testing on core biopsy specimens versus surgical specimens was also utilized to determine which patients were more suitable for neoadjuvant chemotherapy (NCT) or NET. Telemedicine allowed a pathway where the doctor-patient encounter could occur without additional COVID-19 risks, but could it be successfully implemented with a new breast cancer diagnosis?

The American Society of Breast Surgeons (ASBrS) along with other oncologic societies developed guidelines and prioritization for which patients should undergo surgical resection and which patients could have their surgery delayed. ${ }^{2}$ Additionally, the ASBrS rapidly developed a registry for breast cancer patients treated during the COVID-19 pandemic to study alterations in management compared with usual practice. Analyzing the first year of data from this registry, we demonstrated a significant increase in the use of NET for patients with ER+ breast cancer, an increase in the use of genomic testing on the initial core needle biopsy specimen, a limited use for telehealth; and delayed reconstruction and in-patient surgical procedures due to the need to avoid hospitalizations during the initial months of COVID-19. ${ }^{3}$ 


\section{FUTURE}

While use of NET has historically been much higher in the UK than in the USA, the COVID-19 pandemic will likely lead to higher rates of usage of NET both in the USA as well as worldwide. Use of NET has been shown to decrease the size of the primary tumor and permit higher rates of breast conservation. ${ }^{4}$ Additionally, evaluating tumor response to NET, in terms of decrease in tumor size as well as the change in proliferation rate (ki67), can potentially guide the need for chemotherapy. Thus, increasing use of NET, allowing an in vivo assessment of response, may result in a more individualized approach to the use of cytotoxic chemotherapy for breast cancer. The management of patients with breast cancer is a complex multidisciplinary web, where each treatment modality can impact downstream decisions. More importantly, breast cancer is not a single disease but rather a group of diseases with variant tumor biologies and behaviors, and genomic analysis at the time of diagnosis can assist with the complex management decisions. Armed with the knowledge from prior clinical research advances, our guidelines in the treatment of breast cancer were positioned well to adjust to the abrupt conditions created by the pandemic. The modifications we were forced to embrace during the COVID-19 pandemic will likely have a long-lasting impact on breast cancer management and hopefully drive us to a new normal with more individualized and high-value care.

\section{REFERENCES}

1. Park KU, Gregory M, Bazan J, Lustberg M, et al. Neoadjuvant endocrine therapy use in early stage breast cancer during the covid-19 pandemic. Breast Cancer Res Treat. 2021;188(1):249-58. https://doi.org/10.1007/s10549-021-06153-3

2. Dietz JR, Moran MS, Isakoff SJ, et al. Recommendations for prioritization, treatment, and triage of breast cancer patients during the COVID-19 pandemic. The COVID-19 pandemic breast cancer consortium. Breast Cancer Res Treat. 2020;181(3):487-497

3. Wilke LG, Nguyen T, Yang Q, et al. Analysis of the impact of the COVID-19 pandemic on the multidisciplinary management of breast cancer: review from the American Society of Breast Surgeons COVID-19 and Mastery registries. Ann Surg Oncol. 2021. https://doi.org/10.1245/s10434-021-10639-1

4. Olson JA Jr, Budd GT, Carey LA, et al. Improved surgical outcomes for breast cancer patients receiving neoadjuvant aromatase inhibitor therapy: results from a multicenter phase II trial. $J$ Am Coll Surg. 2009;208(5):906-16.

Publisher's Note Springer Nature remains neutral with regard to jurisdictional claims in published maps and institutional affiliations. 